\title{
Estrategias de aprendizaje utilizadas por los estudiantes del 5to año de Educación Media General en el contenido de electromagnetismo
}

\author{
Learning strategies used by the students of the 5th year of General Middle
} Education in the content of electromagnetism

Recibido octubre 2019 Arbitrado noviembre 2019 Publicado enero 2020

\section{Eliuz Katerine Villamizar Rodríguez \\ kattyvillamizar@gmail.com}

Código ORCID: 0000-0003-1265-7950

Unidad Educativa Nacional Bolivariana Paramaconi II, Venezuela

\section{Resumen}

El objetivo general de dicha investigación consistió en analizar las estrategias de aprendizaje utilizadas por estudiantes del 5to año de Educación Media General en el contenido del electromagnetismo. El estudio estuvo enmarcado bajo una modalidad descriptiva, con diseño de campo no experimental. Con una población de 100 estudiantes de quinto año y una muestra intencional de 30 estudiantes que fueron tomados de la sección "A y B". Para la recolección de la información se aplicó un instrumento tipo cuestionario de 4 alternativas conformado por 20 ítems tipo cerrado de selección simple. La confiabilidad se calculó a través del coeficiente de Kuder-Richarson, el cual fue de 0,822; considerado alto. La información permitió evidenciar que los estudiantes no poseen los conocimientos suficientes para el aprendizaje del electromagnetismo y por ende es necesario recomendar relacionar el contenido del contexto con la vida cotidiana del estudiante ya que necesitan los aportes suficientes para el mismo.

\section{Abstract}

The general objective of this research was to understand the learning strategies used by students of the 5th year of General Secondary Education in the content of electromagnetism. The study was framed under a descriptive modality, with a non-experimental field design. With a population of 100 fifth-year students and an intentional sample of 30 students that were taken from section "A and B". To collect the information, a questionnaire-type instrument with 4 alternatives was applied, consisting of 20 closed-type items with simple selection. Reliability was calculated through the Kuder-Richarson coefficient, which was 0.822; considered high. The information made it possible to show that students do not have sufficient knowledge to learn electromagnetism and therefore it is necessary to recommend relating the content of the context with the student's daily life since they need sufficient contributions for it.
Palabras clave:

Estrategias; aprendizaje; electromagnetismo

Keywords:

Strategies; learning; electromagnetism 


\section{INTRODUCCIÓN}

$\mathrm{D}$ esde hace años a nivel mundial en las instituciones educativas, los estudiantes presentan dificultades para comprender los fenómenos de la física, debido a que el docente se dedica únicamente a desarrollar la parte teórica y como única estrategia utiliza el pizarrón, no incentivan al estudiante y lo sobrecarga de contenido, no se percatan del corto tiempo que tienen para explicarlo, excluyendo la parte experimental ya sea por no estar capacitado el docente o por escases de equipos y laboratorios en las instituciones educativas . En el área de la ciencia, las prácticas de laboratorio permiten el estudio de los fenómenos y comprueban la teoría con la experimentación y observan el interés de los estudiantes por la física.

En Venezuela se ha experimentado que existen muchas dificultades en cuanto al aprendizaje de la física, el sistema educativo venezolano sigue enfrentando problemas por los organismos públicos que no se encargan de dotar a los colegios de equipos, aunque existen casos donde estos existen y por falta de seguridad estos no son usados por miedo al robo de los mismos, la ausencia de laboratorios y docentes especialistas en el área de física.

La didáctica de la física, como ciencia experimental, trata de integrar conocimientos que no sean mecánicos ya que la educación es un proceso multidireccional el cual transmite conocimientos, valores, costumbres y formas de actuar.

Aunado a ello, las estrategias didácticas son el conjunto de actividades, técnicas y medios que se planifican de acuerdo con las necesidades de la población a la cual van dirigidas, que puedan ser utilizadas por los docentes para lograr un aprendizaje significativo.

A su vez Ausubel y Colbs (1990), señalan que "la educación es el conjunto de conocimientos, órdenes y métodos por medio de los cuales se ayuda al individuo en el desarrollo y mejora de las facultades intelectuales, morales y físicas. La educación no crea facultades en el educando, sino que coopera en su desenvolvimiento y precisión" (p. 35).

Según dichos autores la educación permite que el estudiante esté en un proceso permanente de construcción, donde considera las experiencias educativas y esto le facilita el desarrollo de conocimiento, destreza, actitudes, valores y habilidades en la física, en el contenido del electromagnetismo, expresan sus leyes por medio 
de las distintas fórmulas y procedimiento, allí el docente deberá implementar nuevas estrategias para atraer la atención del estudiante así poder llegar a obtener un aprendizaje significativo.

Díaz (2010) define una estrategia de aprendizaje como: "un conjunto de pasos o habilidades, y al mismo tiempo un instrumento psicológico que un alumno adquiere y emplea intencionalmente como recurso flexible, para aprender significativamente y para solucionar problemas" (p. 180).

Para el autor antes mencionado, las estrategias de aprendizaje son como pasos o acciones para poder ser aplicado en el momento de la clase, toda estrategia requiere ser adaptada y situada dentro de circunstancias particulares, la idea fundamental no es simplemente que el estudiante repita la información aprendida, sino ir más allá mediante de la elaboración y organización de un contenido.

El electromagnetismo al igual que otros descubrimientos es basado en conceptos de la física haciendo de la vida de los individuos más fácil por ser una fuente de desarrollo para los países de la sociedad y la educación.

En la actualidad las personas utilizan la electricidad y el magnetismo en su día a día, ya que se ha vuelto indispensable para la sociedad y genera varios tipos de energía, para alimentar el consumo del ser humano como el calor, luz y movimiento, son esenciales para la sociedad, en cualquier parte donde se encuentre es acompañado de un constante flujo de energía eléctrica en la casa, ciudad, trabajo y en el quehacer cotidiano.

La gran mayoría de las personas sean niños, adolescentes o adultos han realizado pequeños circuitos y experimentado con imanes, teniendo en cuenta que todo a su alrededor posee electricidad que hace funcionar las cosas en estrecha relación con el magnetismo.

Cabe destacar que a pesar de las preocupaciones y esfuerzos de los docentes e investigadores, de alguna manera la deserción de los estudiantes puede ser por temor a la física o el desinterés porque ven la materia de forma monótona, de ésta manera lo que plantea Piaget (1972) "El gran fallo de las escuelas tradicionales ha sido haber descuidado casi sistemáticamente la formación de los alumnos en las experimentaciones" (p.22).

En consecuencia, lo primordial es plantear la experimentación guiada con materiales comunes y de fácil acceso. Pues, el fin de esta es ser utilizada y realizar la práctica en las aulas de clase para que 
los estudiantes descubran y aprendan algunos fenómenos físicos. A su vez el estudiante observa, relaciona y comprende el porqué de cada fenómeno y así poder relacionar su nuevo conocimiento con lo antes vivido en su día a día tomando en cuenta que la física está en todo lo que nos rodea.

Asimismo, Inciarte et al. (2009) señala que:

En la gestión educativa el docente cumple el papel de orientar e incentivar a los estudiantes para que desarrollen competencias, con capacidades para interiorizar los diferentes elementos que intervienen en el proceso educativo; así el docente como mediador facilita la interacción para que el grupo participe en actividades de análisis sustentadas en una acción reflexiva sobre lo realizado y lo que se puede ejecutar. (p.45)

A partir de lo anterior, entonces el docente debe conocer nuevas estrategias para aplicar en el proceso de aprendizaje de la asignatura de Física en el contenido del electromagnetismo, y de tal manera conocer como captar e incentivar a los estudiantes y descubrir cómo asumen el rol que tienen dentro de dicho proceso, interiorizan los conocimientos impartidos, y los aplican en la vida diaria.

No obstante, el Ministerio del Poder Popular para la Educación ha incorporado cambios en el modelo Educativo Bolivariano de Educación Media General (2016), con el propósito de acercarse a las nuevas exigencias que existen en estos nuevos tiempos. Por lo que propuso en ésta área, los procesos matemáticos y su importancia en la comprensión del entorno.

Sin embargo, muchos docentes se apegan a la enseñanza tradicional ya sea por no estar capacitado, por estar apegado a la rutina y a la educación clásica, en la mayoría de los casos los docentes que están en el área de la física son de matemática donde hay que recordar que dicha materia es una ciencia exacta y no experimental como lo es el caso de la física, y no intentan aplicar estrategias innovadoras que incentiven a los estudiantes a interesarse por el aprendizaje del electromagnetismo.

Por todo lo antes expuesto surge la siguiente interrogante: ¿Cuáles son las estrategias de aprendizaje utilizadas por los 
estudiantes del 5to año de educación media general en física en el contenido del electromagnetismo?

Para alcanzar y dar respuesta a la pregunta objeto de investigación se planteó el siguiente objetivo general: Analizar las estrategias de aprendizaje utilizada por los estudiantes del 5to año de educación media general en la física en el contenido del electromagnetismo.

Buscando dar respuesta al objetivo general se plantearon los objetivos específicos siguientes: 1. Indagar las estrategias de aprendizaje de representación utilizadas por los estudiantes del 5to año de educación media general en la física en el contenido del electromagnetismo. 2. Identificar las estrategias de aprendizaje de concepto utilizada por los estudiantes del 5to año de educación media general en la física en el contenido del electromagnetismo. 3. Determinar las estrategias de aprendizaje de proposiciones utilizada por los estudiantes del 5to año de educación media general en la física en el contenido del electromagnetismo.

Finalmente, esta investigación pretendió mejorar el nivel de conocimiento hacia la física en el contenido del electromagnetismo, puesto que la misma esbozó estrategias de aprendizaje, con la intencionalidad de despertar el interés y motivación de los estudiantes.

\section{Antecedentes}

Los antecedentes se refieren a los estudios realizados por otros autores que sirven de apoyo y sustentan a la investigación teniendo relación con el tema planteado, así como lo comenta Arias (2012), que los mismos "reflejan los avances y el estado actual del conocimiento en un área determinada y sirven de modelo o ejemplo para futuras investigaciones" (p.106).

En el siglo XXI, Venezuela ha tenido avances tecnológicos y científicos en donde aumentan las competencias en las disciplinas de las ciencias experimentales, y en las instituciones lo que está sucediendo es que al entrar el docente en un aula de clases se encuentra con el estudiante que no quiere ver su clase, pero es por la misma razón de no implementar nuevas estrategias de aprendizaje y así poder captar la atención del estudiante por la física en el contenido del electromagnetismo. 
Por esta razón se tiene que crear e implementar nuevas estrategias de aprendizaje en su trabajo diario y así lograr despertar el interés del estudiante en la asignatura de la física en el contenido del electromagnetismo y demostrarle que no es solo una asignatura, sino que la física lo podemos encontrar en todo aquello que nos rodea.

Para aprender los fenómenos eléctricos y magnéticos es necesario recordar unos conceptos tales como: la carga eléctrica, forma de electrización de los cuerpos, estructura eléctrica de la materia, atracción y repulsión, circuitos eléctricos, entre otros. Con ello podemos hacer demostraciones en el aula, realizar montajes de experimentos sencillos que no se requieran de uso de laboratorio.

Tomando en cuenta el estudio de esta investigación se presentan a continuación los trabajos de valiosos investigadores, los cuales aclaran algunos puntos donde apoyan, sustentan y dan un aporte a la investigación planteada porque guardan relación con el tema a desarrollar.

Ortiz (2017) en su estudio denominado "Los experimentos como alternativa de enseñanza y aprendizaje en el magnetismo en estudiantes de 5to año de educación media general de la E.B Vicente Emilio Sojo" enfatizó en la problemática del bajo índice académico y desinterés de la asignatura de física, por lo cual surgió proponer los experimentos como alternativa de enseñanza y aprendizaje del magnetismo, teniendo como base el aprendizaje significativo de Ausubel (1983) en el subsistema de educación, siendo esta una herramienta que sirve de apoyo instruccional para el docente en el desarrollo de las clases para obtener un verdadero aprendizaje de la física y a su vez elevar el índice académico de los estudiantes.

El aporte de este trabajo guardó relación con la presente investigación, ya que se utilizan estrategias de aprendizaje en el contenido de electromagnetismo donde desarrolla la creatividad del estudiante y pueda ver la física de manera didáctica logrando captar la atención del mismo y así poder obtener un aprendizaje significativo.

Por su parte, López (2016), en su trabajo titulado "Estrategia metodológica para mejorar el aprendizaje del electromagnetismo a través del uso de experimentos demostrativos", concluye, a partir del estudio de la factibilidad de las estrategias metodológicas, en diseñar una propuesta para mejorar el aprendizaje del electromagnetismo a 
través del uso de experimentos demostrativos dirigidos a los docentes de la institución.

La investigación mencionada fue de gran importancia para este estudio, porque está orientada a la realización de actividades experimentales demostrativas donde se demuestra la creación de nuevas estrategias para la comprensión en la física en el contenido de electromagnetismo y así poder obtener un mejor aprendizaje.

Morillo y Torres (2016) en su trabajo titulado "Descripción de los conocimientos que poseen los estudiantes acerca del contenido de electromagnetismo en el 5to año de educación media general de la U. E Moral y Luces", se planteó como objetivo general: describir los conocimientos que presentan los estudiantes acerca del contenido de electromagnetismo. Los autores de dicha investigación pudieron evidenciar que los conocimientos que poseen los estudiantes para el aprendizaje del contenido de electromagnetismo son insuficientes, llegando a la conclusión que muchos aparatos eléctricos que incluso se tienen en la casa funcionan gracias a este fenómeno que ha sido tan estudiado por tantos años y que cada vez se presentan nuevos avances en la tecnología, en las comunicaciones gracias al electromagnetismo.

Este trabajo es importante, ya que aporta las bases teóricas del electromagnetismo que requiere la aplicación de nuevas estrategias para poder tener un aprendizaje significativo y así lograr distinguir la física en su forma experimental, donde dichos autores nos explican que el electromagnetismo se encuentra ligado a todo lo que está a nuestro alrededor.

Parra (2017) en su estudio "Estrategias metodológicas utilizadas por los docentes del tercer año de educación media general para la enseñanza del contenido movimiento rectilíneo uniformemente variado bajo el enfoque de Díaz y Hernández", concluye que, aunque existe un número considerable de docentes que afirman conocer estas estrategias, solo un porcentaje menor a la mitad realmente las conocen, lo que resulta contradictorio por encontrarse bajo una educación que centra sus enseñanzas en la obtención de competencias, motivo por el cual los procesos de enseñanza y de aprendizaje se ven afectados y el estudiante no logra relacionar el contenido físico con las necesidades personales y del entorno.

Dicho trabajo es relevante para esta investigación porque, a través de los resultados donde el docente no aplica nuevas estrategias, el estudiante se ve afectado por no tener algo que lo 
incentive a ser creativo y así poder relacionar los contenidos de la física, el docente debería de aplicar nuevas estrategias de aprendizaje que impulsen al mismo a mejorar la calidad de rendimiento académico.

Es necesario destacar que existen algunas estrategias generales $\mathrm{y}$ otras específicas propiamente cognitivas que facilitan el interdominio en relación al aprendizaje de la física en el contenido del electromagnetismo incluyendo habilidades en toma de notas, resumen y observaciones en el momento de enfrentarse con el problema.

De tal manera, radica en el nivel de profundidad que se establezca un aprendizaje. También puede distinguirse entre elaboración visual y verbal-semántica (estrategia de "parafraseo", elaboración inferencial o temática). Evidentemente, estas estrategias permiten un tratamiento y una codificación más sofisticada de la información por aprender, porque atienden básicamente a sus significados y no a sus aspectos superficiales. Díaz Barriga y Hernández (2010, p. 184).

Es importante destacar que la idea fundamental de la aplicación de estrategias de aprendizaje es ir más allá de la información aprendida, donde el estudiante debe descubrir y construir su propio significado cognitivo y así encontrar sentido a la información y mejorar su calidad de conocimiento.

De igual manera lo que se quiere obtener es que el aprendizaje que se produzca sea significativo que relacione el nuevo conocimiento con lo antes vivido, para ello se debe prestar atención a todos y cada uno de los factores que le afecten al estudiante, el papel del lenguaje del docente como facilitador en la física es evidente y tiene que ser receptivo y así poder llegar a la estructura cognitiva del mismo.

De esta forma, actualmente en el proceso de transformación en educación media se establece que el desarrollo de las potencialidades humanas es un proceso abierto, no tiene límites. Refiere a nuestras capacidades para vivir, pensar, sentir, percibir, actuar, transformar, disfrutar, crear, producir, construir... supone entonces la creación de un punto de experiencias, aprendizajes, acciones, situaciones que permitan el desarrollo de estas potencialidades.

Por esta razón, el desarrollo y el avance de la educación venezolana demanda de manera obligatoria la reorientación de las 
estrategias de aprendizaje en el área de física específicamente en el contenido del electromagnetismo, por parte de los docentes debido a que la finalidad educativa debe orientarse hacia un aprendizaje significativo en los estudiantes.

Asimismo, el proceso de transformación en educación media establece que la orientación del proceso de transformación curricular privilegia estos aprendizajes para la vida y la ciudadanía por sobre la preparación para estudios posteriores. No obstante, en la perspectiva de la educación a lo largo de toda la vida, se valoran también aquellos aprendizajes y experiencias que puedan facilitar estudios posteriores, conscientes de que, a partir de la finalización de los niveles obligatorios de la educación, se abren caminos diversos a nuestras y nuestros jóvenes.

De aquí la importancia para el estudiante conocer los diferentes tipos de estrategia aplicadas en su aprendizaje, ya que esto le ayuda a internalizar el conocimiento dado por el docente y construir esquemas de habilidades que le permitan adquirir una visión de transformación humana del saber, a su vez se obtiene un mejor aprendizaje en el área de física en el contenido de electromagnetismo.

Los estudios referenciales invitan a estimar lo que Ausubel (1983) sostiene sobre el aprendizaje significativo:

El aprendizaje significativo basado en la recepción supone principalmente la adquisición de nuevo significado a partir del material de aprendizaje presentado. Requiere tanto de una actitud de aprendizaje significativo como la representación al estudiante de un material potencialmente significativo... la interacción entre significados potencialmente nuevos e ideas pertinentes en las estructuras cognitivas del estudiante da lugar a significados reales o psicológicos. Puesto que la estructura cognitiva de cada persona que aprende es única, todos los nuevos significados adquiridos también son forzosamente únicos. (p.26)

De acuerdo con lo que dice el autor, el estudiante relaciona un nuevo conocimiento con lo que ya posee y así construye su propio concepto mediante las experiencias vividas, en la actualidad el docente solo llega al aula de clase y utiliza como estrategia la pizarra y no se da cuenta de las consecuencias que esto ocasiona en el rendimiento y afectividad de la educación hacia esta ciencia 
experimental el docente no se percata lo fácil que es demostrar todos los contenidos relacionados con la física ya que lo puede relacionar con la vida cotidiana.

Al respecto, Díaz y Hernández (2002), indican lo siguiente:

Una estrategia de aprendizaje es un procedimiento (conjuntos de pasos y habilidades) que un alumno adquiere y emplea de forma tradicional como instrumento flexible para aprender significativamente y solucionar problemas y demandas académicas. Los objetivos particulares de cualquier estrategia de enseñanza pueden consistir en afectar la forma en que se selecciona, adquiere, organiza o integra el nuevo conocimiento, o incluso la modificación del estado afectivo o motivacional del aprendiz, para que este aprenda con mayor eficacia los contenidos curriculares y extracurriculares que se presentan. (p.70)

Según Díaz y Hernández es importante destacar que el docente especialista debe conocer su propósito y ser capaz de analizar y generar nuevas estrategias de aprendizaje e impartir la información de manera que le llegue al estudiante de una forma clara y sencilla facilitando el dominio y comprensión en el contenido del electromagnetismo y así demostrar su utilidad en la vida cotidiana.

\section{MÉTODO}

$\mathrm{E}$ l tipo de investigación se ubica en un trabajo descriptivo, ya que se compila la información específica, de grupos, persona, o comunidades, se amplía la información y se da las respuestas a las preguntas de la investigación.

Según Arias (2012) "la investigación descriptiva consiste en la caracterización de un hecho, fenómeno, individuo o grupo, con el fin de establecer su estructura o comportamiento. Los resultados de este tipo de investigación se ubican en nivel intermedio en cuento a la profundidad de los conocimientos se refiere" (p.24).

Por otra parte, es preciso expresar que la investigación se fundamentó bajo un diseño de campo, con los cuales se permitió la recolección de datos de sujetos obtenidos de la realidad sin manipular ni alterar las condiciones existentes. 
Los datos fueron recolectados de manera directa a estudiantes, a través de un instrumento se extrajo la información, se analizaron los datos, y se observó el conocimiento de los mismos acerca de la asignatura de física en el contenido de electromagnetismo en el 5to año de Educación Media General de la Unidad Educativa Nacional Bolivariana Paramaconi II.

Cabe destacar que para llevar a cabo la investigación se utilizó una población de cien (100) estudiantes del 5to año de Educación Media General de la Unidad Educativa Nacional Bolivariana Paramaconi II, distribuidos de la siguiente manera en la sección A 24 en la sección B 25 en la sección C 25 y en la sección D 26.

Según Arias (2012), la muestra es un subconjunto representativo y finito que se extrae de la población accesible (p.83). Cabe destacar que en dicha investigación se seleccionaron como muestra del estudio a los estudiantes del 5to año de la Unidad Educativa Nacional Bolivariana Paramaconi II, los cuales resultaron treinta (30) estudiantes, seleccionados de la siguiente manera: de la sección B (25) estudiantes, y de la sección A (5) estudiantes, los mismos fueron seleccionados para el desarrollo de la investigación.

El instrumento que se empleó para recolectar la información fue el cuestionario que consta de una serie de preguntas en relación con el contenido de dicho estudio. El instrumento fue elaborado por Angulo y Adreli (2012), para la investigación Estrategia de aprendizaje del contenido del electromagnetismo dirigidos a estudiantes de quinto año de la Unidad Educativa "Luis Sanojo", el cual consta de veinte (20) ítems de preguntas abiertas de selección simple, de cuatro alternativas, formulando las preguntas en función de describir los conocimientos acerca del electromagnetismo en quinto año.

Para estimar la confiabilidad en dicha investigación se utilizó el SPSS.18 el coeficiente de Kuder-Richarson (KR-20), en el siguiente estudio obtuvo un índice de 0,822 , lo cual es importante decir que el instrumento es confiable. También puede interpretarse que el instrumento se puede aplicar en varias ocasiones con distintos estudiantes y arrojará resultados similares, lo que resulta un grado alto de confiabilidad.

Según Tamayo y Tamayo (2006), expresa que "el análisis de los datos tiene su significado únicamente, en función de las interpretaciones que obtenga el investigador, que ha sometido a un proceso analítico los resultados obtenido mediante técnicas 
estadísticas" (p.75). Una vez culminada la fase de recolección de datos se inicia con la codificación de la formulación y su tabulación mediante los datos contenidos en el instrumento, se analizó mediante el programa de Excel de office y el paquete estadístico Estatistical Packegfor Social Gerencia (SPSS18), con la cual se calculó la variable en función a las respuestas obtenidas.

\section{RESULTADOS Y DISCUSIÓN}

$\mathrm{E}$ n las notas de campo llevadas por la investigadora durante la ejecución de la técnica de la observación, se pudo resumir que:

En el caso de los estudiantes del Quinto Año Sección "B" de Educación Media General de la Unidad Educativa Nacional Bolivariana Paramaconi II, ubicado en Chirgua Sector la Colonia Del Municipio Bejuma Parroquia Simón Bolívar del estado Carabobo, los mismos han presentado dificultad en la comprensión y ejecución en la asignatura de Física, debido a la falta de aplicación de estrategias de aprendizaje, que capten el interés de los estudiante. Esto trae como consecuencia lo siguiente:

- Bajos resultados de las evaluaciones.

- Desinterés presentado durante las clases.

- Incomprensión de los enunciados.

- Manejo de poca cantidad de términos científicos.

- Uso de técnicas inadecuadas.

- Además, la poca interpretación y comprensión del contenido electromagnetismo.

- Poco interés en la realización de otros experimentos apartes de la elaboración del circuito, que es el único experimento realizado en el año escolar.

Debido a lo antes expuesto, se puede decir que los docentes no aplican las estrategias de aprendizaje en el contenido del electromagnetismo a los estudiantes del 5to año de educación media general aun conociendo que lo que se quiere alcanzar es un aprendizaje constructivo y significativo que conlleve al estudiante a ver la asignatura de física de una manera no tradicional sino como una ciencia experimental. 
Por otra parte, los planteamientos, de dicho estudio parte de las estrategias de aprendizaje utilizada por los estudiantes del 5to año de Educación Media General en la Unidad Educativa Nacional Bolivariana Paramaconi II, donde se analizaron y se obtuvieron evidencias que permitieron plantear lo siguiente:

El contenido del "electromagnetismo", se puede decir que muchos aparatos eléctricos en la casa funcionan gracias a este fenómeno y que día a día va avanzando la tecnología, en las comunicaciones se puede observar como dos fenómenos como la electricidad y el magnetismo están ligados un solo contenido, que a través de los años ha sido material de estudio para muchos investigadores.

Por otro lado, se evidenció que en el primer objetivo solo un $60 \%$ de los estudiantes encuestados en la investigación, lograron identificar el campo magnético el cual es un fenómeno físico a el que ejerce fuerzas de atracción ante algunos objetos, el níquel, el cobalto el hierro y las aleaciones se les llama imanes. Y un $20 \%$ presentan dificultar para entender el aprendizaje del tema.

En el segundo objetivo un 32\% de los estudiantes logran conocer el contenido de la Ley de Ampere, y reconocen las formulas y las pueden aplicar en los campos magnéticos con las corrientes. Logrando observar que un $18 \%$ de los estudiantes no reconocen dichas formulas y les cuesta parte conceptual y que no reconocen la ley de Biot y Savart.

En tercer objetivo, un 22\% logra tener conocimiento en lo que es la corriente inducida. De igual manera es necesario para que el estudiante comprenda como se produce la inducción ya que de esta corriente se produce la inducción electromagnética. La corriente eléctrica inducida surge en un conductor en reposo dentro de un campo magnético variable con el tiempo o en un conductor que se mueve dentro de un campo magnético estacionario, de tal modo.

En el cuarto y último objetivo, un $16 \%$ de los estudiantes encuestados tienen conocimiento acerca de los aparatos eléctricos y electrónicos y que son alimentados con corrientes alternas el cual se produce al hacer girar una bobina con movimiento circular uniforme en un campo magnético homogéneo.

Finalmente es importante mencionar que todo lo que nos rodea tiene electricidad tanto en los hogares como en el campo industrial eso es de gran utilidad en el momento de explicar el contenido del 
electromagnetismo y así al docente se le facilita la explicación tomando como ejemplo su vida diaria.

\section{CONCLUSIONES}

0 e presentan estas líneas finales a partir de lo expresado por Díaz y Hernández (2010), pues para que los estudiantes puedan comprender los contenidos del electromagnetismo, los docentes deben saber utilizar ciertos recursos y estrategias que faciliten el aprendizaje tratando de relacionar el contenido con la vida cotidiana del estudiante y así poder pasar los mismos sin ninguna dificultad ya que se puede decir que lo manejan a la perfección.

El docente en el área de la física debe tomar en cuenta los conocimientos del estudiante y así poder perfeccionar el alcance de las competencias, siempre debe recordar bien sus funciones como mediador orientando y guiando la actividad constructivista de sus estudiantes para ello es importante aportar las herramientas necesarias que incluyan estrategias de aprendizaje para despertar el interés del mismo de la materia experimental, así como lo es la física.

El propósito del docente debe ser promover mediante estrategias de aprendizaje la física en su forma experimental antes o durante la clase para obtener buenos resultados en el estudiante, el profesor debe desarrollar estrategias que ayuden a los mismos a adquirir nuevo conocimiento educativo con el fin de lograr que sea creativo y capaz de analizar y asimilar la información.

\section{REFERENCIAS}

Arias, F. (2012). El proyecto de investigación. (6ta Ed.). Caracas: Episteme

Ausubel y Colbs. (1990). Psicología Educativa: Un punto de vista cognoscitivo. México: Trillas

Ausubel, Novac, Hanesiar. (1983). Psicología Educativa, Un punto de vista cognitivo. México: Trillas

Díaz, F. y Hernández, G. (2010). Estrategias docentes para un aprendizaje significativo. Una interpretación constructivista. México: Mc Graw Hill

Díaz, F. y Hernández, G. (2002). Aportaciones de las perspectivas constructivistas y reflexivas en la formación docente en el bachillerato. México: Mc. Graw Hill 
Inciarte y otros. (2009). Enseñanza de las ciencias y la matemática. Organización de Estados Iberoamericanos para la Educación, la Ciencia y la Cultura. Recuperado de www.oei.org.co/oeivirt/edumat

López, Y. (2016). Estrategia metodológica para mejorar el aprendizaje del electromagnetismo a través del uso de experimentos demostrativos (Trabajo de Grado de Maestría) Universidad de Carabobo, Venezuela

Morillo y Torres. (2016). Descripción de los conocimientos que poseen los estudiantes acerca del contenido de electromagnetismo en el 5to año de educación media general de la U.E Moral y Luces (Trabajo de Grado). Universidad de Carabobo, Venezuela

Ortiz, G. (2017). Los experimentos como alternativa de enseñanza y aprendizaje en el magnetismo en estudiantes de 5to año de educación media general de la E.B Vicente Emilio Sojo. (Trabajo de Grado de Maestría) Universidad de Carabobo, Venezuela

Parra, S. (2017). Estrategias metodológicas utilizadas por los docentes del tercer año de educación media general para la enseñanza del contenido del contenido movimiento rectilíneo uniformemente variado bajo el enfoque de Díaz y Hernández (Trabajo de Grado) Universidad de Carabobo, Venezuela

Piaget, J. (1972). Psicología y Epistemología. Barcelona: Ariel Sabino, C. (2007). El Proceso de Investigación. Caracas: Panapo Tamayo, M. y Tamayo, F. (2006). Proceso investigativo. México: Prentice Hall 